\title{
Atividade anti-helmíntica do fruto da Melia azedarach em cordeiros naturalmente infectados com nematódeos gastrintestinais
}

\section{Anthelmintic activity of Melia azedarach fruits in lambs naturally infected with gastrointestinal nematodes}

\author{
Margarete Kimie Falbo ${ }^{1 *}$; Itacir Eloi Sandini²; Helcya Mime Ishiy ${ }^{3}$; \\ Jorge Luis Fávaro ${ }^{2}$; Carlos Eduardo dos Santos ${ }^{4}$; Sinthia Bastos ${ }^{4}$; \\ Daniel Rodigheri'; Daniela Guzzo ${ }^{4}$
}

\section{Resumo}

Objetivou-se neste trabalho avaliar a atividade anti-helmíntica dos frutos secos e moídos da planta Melia azedarach, em cordeiros naturalmente infectados com nematódeos gastrintestinais. Utilizou-se 24 cordeiros, com 90 dias de idade, machos, mestiços Ile de France x Corridale, os quais foram divididos em 3 grupos de oito animais cada, sendo: grupo 1, medicado com $5 \mathrm{mg} \mathrm{Kg}^{-1} \mathrm{em}$ dose única, de albendazole pela via oral (V.O); grupo 2, com $2 \mathrm{~g} . \mathrm{Kg}^{-1}$ (V.O), em dose única, do fruto do cinamomo (Melia azedarach) e o grupo 3, controle, não recebeu nenhum tipo de tratamento. Os resultados encontrados demonstraram que o grupo tratado com albendazole foi o que apresentou melhor eficácia $51.96 \%$, seguido do grupo tratado com frutos de cinamomo o qual apresentou eficácia de 33.21\%.

Palavras-chave: Melia azedarach, fitoterapia, verminose, cordeiros

\begin{abstract}
The aim of this study was to evaluate the anthelmintic activity of Melia azedarach grounded dry fruits, in lambs naturally infected with gastrointestinal nematodes. Twenty four (24) mixed-breed Ile de France $x$ Corriedale, male lambs, aged 3 month, were separeted into three (3) groups of eight (8) animals: the first group was medicated with $5 \mathrm{mg} . \mathrm{Kg}^{-1}$ of albendazole orally, in a single dose, the second group was medicated with $2 \mathrm{~g} . \mathrm{Kg}^{-1}$ of Melia azedarach fruits orally, in a single dose, and the third group remained untreated, as control. The results showed that the group that presented the best efficacy was the one treated with albendazole (51.96\%), followed by the group treated with Melia azedarach fruits that presented $33.21 \%$ of efficacy.
\end{abstract}

Key words: Melia azedarach, phytoterapy, gastrointestinal nematodes, lambs

\footnotetext{
1 Docente do Departamento Medicina Veterinária da Universidade Estadual do Centro Oeste, UNICENTRO, Guarapuava-PR. E-mail: margaretefalbo@hotmail.com.

2 Docentes do curso de Agronomia da Universidade Estadual do Centro Oeste, UNICENTRO, Guarapuava-PR.

3 Docente do Departamento Medicina Veterinária da Universidade Estadual do Centro Oeste, UNICENTRO, Guarapuava-PR

4 Acadêmicos do curso Medicina Veterinária da Universidade Estadual do Centro-Oeste, UNICENTRO, Guarapuava-PR.

* Autor para correspondência
} 


\section{Introdução}

O parasitismo por nematódeos gastrintestinais tem se constituído num dos principais fatores limitantes à exploração de caprinos e ovinos no Brasil (PADILHA, 1980). Ocontrole desses parasitosnestas espécies animais vem sendo realizado através do uso de anti-helmínticos pertencentes a diversos grupos químicos, na maioria das vezes, sem considerar os fatores epidemiológicos predominantes na região, os quais interferem diretamente na população parasitária ambiental e, consequentemente, na infecção do rebanho.

Desde a década de 90 vem se alertando que o uso intensivo dos compostos químicos é o fator mais importante para o aparecimento da resistência parasitária (THOMAZ-SOCCOL et al., 1996). Exemplo disto é a resistência dos parasitos aos antihelmínticos nos três Estados da região Sul: Paraná, em que alguns rebanhos já não dispõem mais de princípio ativo eficaz (THOMAZ-SOCCOL et al., 1996, 2004); Rio Grande do Sul onde a resistência à ivermectina e aos benzimidazóis atingia 13\% e 90\% respectivamentedas propriedades(ECHEVARRIAet al., 1996) e em Santa Catarina onde vários princípios ativos de anti-helmínticos foram assinalados com resistência (ROSALINSKI-MORAES et al., 2007).

Melo et al. (2003) em um estudo realizado no estado do Ceará, verificaram que a prevalência de nematódeos resistentes a oxfendazol, levamisol e ivermectina em ovinos foi de $88 \%, 41 \%$ e $59 \%$, e em caprinos de $87.5 \%, 75 \%$ e $37.5 \%$, respectivamente. Observaram também que o gênero Haemonchus foi o mais prevalente na população resistente a todos os anti-helmínticos, tanto em ovinos quanto em caprinos, seguido pelo gênero Trichostrongylus e Oesophagostomum.

Em virtude desta situação vários estudos têm sido realizados na busca de alternativas para o controle da verminose em pequenos ruminantes, dentre eles, destaca-se o controle biológico, redução na freqüência de tratamentos anti-helmínticos, vacinas, seleção de raças mais resistentes a parasitos, e a utilização de plantas com atividades antiparasitárias (WALLER, 1999) que tem sua origem na medicina etnoveterinária (ATHANASIADOU; GITHIORI; KYRIAZAKIS, 2007).

Segundo Chagas (2004) a aplicação de extratos vegetais pode causar um desenvolvimento mais lento da resistência, além de normalmente atingir somente as espécies alvo, serem biodegradáveis, não causarem poluição ambiental e diminuírem drasticamente o problema dos resíduos. As pesquisas têm buscado desenvolver produtos baseados em substâncias naturais, que tenham a capacidade de interferir nos processos biológicos dos parasitos, como reguladores de crescimento e também no comportamento alimentar.

Muitas plantas já têm sido descritas com propriedades anti-helmínticas (VIEIRA et al., 1999; GITHIORI, 2004). Dentre elas, a planta Melia azedarach L., pertencente à família Meliaceae, popularmente conhecida como cinamomo, santa-bárbara, lírio, natural do sul da Ásia, hoje amplamente distribuída em quase todos os países tropicais e subtropicais. No Brasil, possui um cultivo amplo (NAKATANI et al., 1995), principalmente na região Sul do país (DANTAS et al., 2000).

Yamasaki et al. (1988) relataram que os extratos de folhas e sementes do cinamomo contêm cerca de quatro compostos ativos, azadiractina, salanina, meliantrol e nimbim. Segundo Matias et al. (2002) os metabólitos melianol e meliantriol, são triterpenóides presentes no fruto e apresentam atividade anti-helmíntica.

Girão e Carvalho (1999) realizaram um levantamento das plantas conhecidas pelos criadores de caprinos do Piauí com possível efeito antihelmíntico, e muitas delas apresentaram resultados promissores, como a batata-de-purga (Operculina sp) e o cinamomo (M. azedarach).

Akhtar e Riffat apud Iqbal et al. (2003) relataram que a planta $M$. azedarach possui atividade antihelmíntica em caprinos. 
O descobrimento de novos grupos antihelmínticos e outros métodos de controle parasitário é estrategicamente necessário para a preservação da economia rural e a manutenção da saúde dos animais (GEARY; THOMPSON; KLEIN, 1999).

O objetivo deste trabalho foi avaliar a atividade anti-helmíntica dos frutos secos da $M$. azedarach em ovinos, naturalmente infectados com parasitos gastrintestinais.

\section{Material e Métodos}

O experimento foi conduzido na Universidade Estadual do Centro Oeste - Guarapuava/Pr, numa área de 2000 metros quadrados, formada com pastagem de milheto (Penissetum americanum).

Foram utilizados 24 cordeiros naturalmente infectados com helmintos gastrintestinais, machos, com 90 dias de idade, mestiços Ile de France com Corriedale, divididos em 3 grupos, através da randomização do número de ovos por grama de fezes (OPG). O grupo 1 foi tratado com $5 \mathrm{mg} \cdot \mathrm{Kg}^{-1}$ em dose única, de albendazole pela via oral (V.O), o grupo 2, com $2 \mathrm{~g} . \mathrm{Kg}^{-1}$ (V.O), em dose única, do fruto (M. azedarach) e o grupo 3, controle, não recebeu nenhum tipo de tratamento, somente água destilada (V.O.). O fruto do cinamomo seco foi triturado e acrescido de farelo de milho e açúcar mascavo para aumentar a palatabilidade.

Amostras fecais foram colhidas, diretamente do reto, no dia zero e oito dias após os tratamentos, para realização da contagem de ovos por grama de fezes (OPG), os quais foram feitos em triplicata, conforme técnica de Gordon e Whitlock (1939) e, o cultivo de larvas infectantes como descrito por Roberts e O’Sullivan (1950). Para identificação das larvas infectantes, utilizou-se a chave de identificação de Keith (1953).

Utilizou-se o teste de redução de contagem de ovos por grama de fezes conforme metodologia descrita pela World Association Advanced
Parasitology Veterinary (WAAPV) que permitiu estimar a eficácia dos diferentes tratamentos, por comparação da redução da contagem de OPG, antes e após o tratamento, segundo fórmula descrita por Coles e Rousch (1992):

$\%$ da eficácia $=[1-(\mathrm{OPG}$ Grupo medicado $/ \mathrm{OPG}$ no grupo controle)] x 100

Para análise de variância, os dados foram transformados em raiz quadrada $(\mathrm{X}+1)$ e as médias comparadas utilizando o teste de Tukey em nível de $5 \%$ de probabilidade, aplicando o Sistema de Análises Estatísticas -SANEST (ZONTA; MACHADO, 1987).

\section{Resultados e Discussão}

Os resultados encontrados demonstraram que o grupo tratado com albendazole foi o que apresentou melhor eficácia, 51.96\%, seguido do grupo com frutos de cinamomo $33.21 \%$ (Tabela1).

$\mathrm{Na}$ coprocultura, os três grupos demonstraram a predominância do gênero Haemonchus sp. (98.8\%), sendo também observado o gênero Trichostrongylus sp. $(0.12 \%)$.

Quanto ao grupo tratado com albendazole, observou-se que não houve o controle da verminose como esperado, possivelmente devido à resistência dos parasitos ao anti-helmíntico utilizado. Molento (2004), em um estudo realizado em Restinga-RS, com ovinos, verificou resistência a vários princípios ativos, tendo o albendazole apresentado somente $45 \%$ de eficácia, atribuindo este fato ao uso intensivo de drogas associado à facilidade de compra dos medicamentos pelos produtores.

Conforme critérios sugeridos pela World Association for the advancement of Veterinary Parasitology - W.A.A.V.P (COLES et al.,1992), reduções menores que $90 \%$ na contagem de OPG, a droga pode ser considerada moderadamente efetiva e, nesse caso, há suspeita de resistência antihelmíntica. 
O grupo tratado com o fruto de cinamomo também não apresentou eficácia (33.21\%) no controle da verminose. Observou-se também, que mesmo adicionando farelo de milho e açúcar mascavo aos frutos de cinamomo ainda houve alguma resistência à ingestão, demonstrando uma baixa palatabilidade dos frutos. Chagas e Vieira (2007), também observaram palatabilidade negativa em caprinos ao administrar folhas verdes e secas da planta Neem pertencente à mesma família da $M$. azedarach.

Desta forma, concentrações maiores que a utilizada neste trabalho poderia ser inviável não só devido à palatabilidade com também a toxicidade da planta. Segundo Kingsbury apud Mendez et al. (2002), a dose tóxica para ovinos é de aproximadamente $0.5 \%$ do peso vivo. Mendez et al. (2002) verificaram sinais clínicos (atonia ruminal e apatia) em bovinos após a ingestão experimental de $5 \mathrm{~g} . \mathrm{Kg}^{-1}$ de folhas verdes do cinamomo.

Girão e Carvalho (1999) utilizaram frutos secos triturados da planta $M$. azedarach na dosagem de 2 e $3 \mathrm{~g} . \mathrm{Kg}^{-1}$ de peso vivo, via oral, e obtiveram $59 \%$ e $54 \%$ de eficácia anti-helmíntica, respectivamente, em caprinos.

Maciel et al. (2006) testaram à atividade ovicida e larvicida do extrato dos frutos e folhas do cinamomo e verificaram que o extrato dos frutos foi mais ativa sobre ovos de $H$. contortus, e que o extrato das folhas do cinamomo foram mais ativo sobre as larvas do mesmo nematódeo.

Conforme Athanasiadou, Githiori e Kyriazakis (2007) há uma grande variedade de métodos para testar a atividade anti-helmíntica das plantas e isso têm gerado uma variabilidade experimental com resultados conflitantes. Além disso, outros aspectos como biodisponibilidade dos compostos ativos em diferentes partes do trato gastrintestinal, espécie animal utilizada no experimento, variabilidade sazonal e ambiental na composição da planta, estágio de desenvolvimento, processo de secagem, extração e estocagem contribuem em aumentar o grau de variabilidade observados nas pesquisas envolvendo a medicina etnoveterinária.

Sem sombra de dúvidas, muitos estudos serão ainda necessários para a validação das propriedades anti-helmínticas das plantas. Apesar dos resultados obtidos neste estudo não apresentarem satisfatórios no controle da verminose em ovinos, acredita-se que mais pesquisas deverão ser realizadas com a $M$. azedarach utilizando não só o fruto como também outras partes da planta ou, o seu extrato, buscando isolar o(s) composto(s) ativos responsáveis pela ação anti-helmíntica, estudando o mecanismo de ação destes compostos, e, avaliar in vitro e in vivo, qual a melhor forma de administração, dose e posologia, monitorando os efeitos anti-nutricionais e/ou tóxicos da planta.

\section{Agradecimentos}

Ao $\mathrm{CNPq} / \mathrm{MDA}$, pelo financiamento do Projeto "Processo produtivos Agroecológicos para Agricultura Familiar em Sistemas de Redes de Propriedades de Referência", e ao Instituto Agroflorestal Bernardo Hakwoort - IAF/ Turvo-PR. 
Tabela 1. Contagem de ovos por grama de fezes (OPG) no dia zero, oito dias após tratamento e eficácia.

\begin{tabular}{cccc}
\multicolumn{5}{c}{ OPG } \\
\hline Tratamento & Dia Zero & 8 dias & Eficácia (\%) \\
\hline Albendazol & $6630 \mathrm{~ns}$ & $3178 \mathrm{~b}$ & 51,96 \\
Cinamomo & 6326 & $4419 \mathrm{ab}$ & 33,21 \\
Controle & 6498 & $6616 \mathrm{a}$ & ------- \\
\hline Média & 6479 & 5602 & \\
\hline
\end{tabular}

As médias seguidas pela mesma letra minúscula na coluna não diferem estatisticamente entre si ao nível de $5 \%$ de probabilidade pelo teste de Tukey. ns: não significativo

\section{Referências}

ATHANASIADOU, S.; GITHIORI, J.; KYRIAZAKIS, I. Medicinal plants for helminth parasite control: facts and fiction. Animal, Cambridge, v. 1; n. 9; p. 1392-1400, 2007.

CHAGAS, A. C. S. Controle de parasitas utilizando extratos vegetais. Revista Brasileira de Parasitologia, São Paulo, v. 13, n. 1, p. 156-160, 2004.

CHAGAS, A. C. S.; VIEIRA, L. S. Ação de Azadirachta indica (Neem) em nematódeos gastrintestinais de caprinos. Brazilian Journal Veterinary Research Animal Science, São Paulo, v. 44, n. 1, p. 49-55, 2007.

COLES, G. C.; BAUER, C.; BORGSTEEDE, F. H. M.; GEERTS, S.; KLEI, T. R.; TAYLOR, M. A.; WALLER, P. J. World Association for the Advancement of Veterinary (W.A.A.V.P.) methods for the detection of anthelintic resistence in nematodes of veterinary importance. Veterinary Parasitology, Amsterdam, v. 44, n. 1, p. 3544, 1992.

COLES, G. C.; ROUSCH, R. T. Slowing the spread of anthelmintic resistant nematodes of sheep and goats in the United Kingdon. Veterinary Record, London, v. 130, n. 23, p. 505-510, 1992.

DANTAS, D. A.; MAGANHA, M.; BERETTA, T. E.; NOZU, P.; PEREIRA, G. S.; MATIAS, R.; SOLON, S.; RESENDE, U.; KOLLER, W. W.; GOMES, A. Estudo fitoquímico dos frutos de Melia azedarach L. (Cinamomo, Meliaceae). In: ENCONTRO DE PESQUISA E INICIAÇÃO CIENTÍFICA DA UNIDERP, 2., 2000, Campo Grande. Anais... Campo Grande: UNIDERP, 2000. p. 119-120.

ECHEVARRIA, F.; BORBA, M. F. S.; PINHEIRO, A. C.; WALLER, P. J.; HANSEN, J. W. The prevalence of anthelmintic resistance in nematodes parasites of sheep in Southern Latin America: Brazil. Veterinary Parasitology, Amsterdam, v. 62, n. 3, p. 199-206, 1996.
GEARY, T.; THOMPSON, D.; KLEIN, R. Mechanismbased screening: discovery of the next generation of anthelmintics depends on more basic research. International Journal for Parasitology, Oxford, v. 29, n. 1, p. 105-112, 1999.

GIRÃO, E. S.; CARVALHO, J. H. Avaliação de plantas medicinais com efeito anti-helmíntico em caprinos. Revista do Conselho Federal de Medicina Veterinária, Brasília, v. 5, n. 18, p. 14-16, 1999.

GITHIORI, J. B. Evaluation of anthelmintic properties of ethnoveterinary plant preparations used as livestock dewormers by pastoralists and small holder farmers in Kenya. 2004. Thesis. (Doctorate Biomedical Sciences and Veterinary Public Health) - Swedish University of Agricultural Sciences, Suécia.

GORDON, H. M.; WHITLOCK, H. N. A new technique for counting nematode egg in sheep faeces. Journal of Council for Scientific and Industrial Research, Melbourne, v. 12, n. 1, p. 50-52, 1939.

IQBAL, Z.; AKHTAR, M. S.; SINDHU, Z.; KHAN, M. N.; JABBAR, A. Review Herbal Dewormers in Livestock - A Traditional Therapy. International Journal of Agriculture \& Biology, Faisalabad, v. 5, n. 2, p. 199206, 2003.

KEITH, R. K. The differentiation of the infective larvae of some common nematode parasites of cattle. Australian Journal of Zoology, Victoria, v. 1, n. 2, p. 223-235, 1953.

MACIEL, M. V.; MORAIS, S. M.; BEVILAQUA, C. M. L.; CAMURÇA-VASCONCELOS, A. L. F.; COSTA, C. T. C.; CASTRO, C. M. S. Ovicidal and larvicidal activity of Melia azedarach extracts on Haemonchus contortus. Veterinary Parasitology, Amsterdam, v. 140, n. 1-2, p. 98-104, 2006.

MATIAS, R.; SOLON, S.; RESENDE, U. M.; GOMES, A.; KOLLER,W. W. Melia azedarach: uso popular x estudos químicos e farmacológicos: breve revisão. Ensaios e Ciência, Campo Grande, v. 6, n. 1, p. 91-121, 2002. 
MELO, A. C. F. L.; REIS, F. I.; BEVILAQUA, C. M. L.; VIEIRA, L. S.; ECHEVARRIA, F. A. M.; MELO, L. M. Nematódeos resistentes a anti-helmíntico em rebanhos de ovinos e caprinos do estado do Ceará. Ciência Rural, v. 33, n. 2, p. 339-344, 2003.

MENDÉZ, M. C.; ARAGÃO, M.; ELIAS, F., RIETCORREA, F.; GIMENO, E. J. Experimental intoxication by the leaves of Melia azedarach (Meliaceae) in cattle. Pesquisa Veterinária Brasileira, Rio de Janeiro, v. 22, n. 1, p. 19-24, 2002.

MOLENTO, M. B. Multidrug resistance in Haemonchus contortus associated with suppressive treatment and rapid drug alternation. In: CONGRESSO BRASILEIRO DE PARASITOLOGIA VETERINÁRIA, 13., 2004, Ouro Preto. Anais... Ouro Preto: Revista Brasileira de Parasitologia Veterinária, 2004. p. 121.

NAKATANI, M.; HUANG, R. C.; OKAMURA, H.; IWAGAWA, T.; TADERA, K. Salannal, a new limonoid from Melia azedarach Linn. Chemistry Letters, Tokyo, v. 24, n. 11, p. 995-996, 1995.

PADILHA, T. N. Doenças parasitárias em caprinos nas regiões áridas e semi-áridas do nordeste brasileiro. Petrolina: Embrapa-CPATSA, 1980. Documento 17.

ROBERTS, F. H. S; O'SULLIVAN, J. P. Methods for egg counts and larval cultures for strongyles infesting the gastrointestinal tract of cattle. Australian Journal of Agriculture Research, Collingwood, v. 1, n. 1, p. 99-102, 1950.

ROSALINSKI-MORAES, F.; MORETTO, L. M.; BRESOLIN, W. S.; GABRIELLI, I.; KAFER, L.; ZANCHET, I. K.; SONAGLIO, F.; THOMAZSOCCOL, V. Resistência anti-helmíntica em rebanhos ovinos da região da associação dos municípios do Alto Irani (Amai), oeste de Santa Catarina. Ciência Animal Brasileira, Goiânia, v. 8, n. 3, p. 559-565, jul./set. 2007.
THOMAZ-SOCCOL, V.; SOTOMAIOR, C.; SOUZA, F. P.; CASTRO, E. A. Occurrence of resistance to anthelmintics in sheep in Paraná State, Brazil. Veterinary Record, London, v. 139, n. 17, p. 421-422, 1996.

THOMAZ-SOCCOL, V.; SOUZA, F. P.; SOTOMAIOR, C.; CASTRO, E. A.; MILCZEWSKI, V.; PESSOA, M. C.; MOCELIN, G. Resistance of gastrointestinal nematodes of anthelmintics in sheep (Ovis aries). Brazilian Archives of Biology and Technology, Curitiba, v. 47, n. 1, p. 41-47, 2004.

VIEIRA, L. S.; CAVALCANTE, A. C. R.; PEREIRA, M. F.; DANTAS, L. B.; XIMENES, L. J. F. Evaluation of anthelmintic efficacy of plants available in Ceara state, North-east Brazil, for the control of goat gastrointestinal nematodes. Revue de Medecine Veterinaire, Toulouse, v. 150, n. 5, p. 447-452, 1999.

WALLER, P. J. International approaches to the concept of integrated control of nematode parasites of livestock. International Journal Parasitology, Oxford, v. 29, n. 1, p. 155-164, 1999.

ZONTA, E. P.; MACHADO, A. A. SANEST: sistema de análises estatísticas para microcomputadores. Pelotas: DMEC/IFM/UFPel, 1987.

YAMASAKI, B. R.; RITLAND, T. G.; BARNBY, M. A.; KLOCKE, J. A. Isolation and purification of salannin from neem seeds and its quantification in neem and chinaberry seeds and leaves. Journal of Chromatography A, Amsterdam, v. 447, p. 217-283, 1988. 